\title{
The Irregularity of the Direct Image of Some $\mathcal{D}$-modules
}

By

\author{
Céline RoucairoL*
}

\begin{abstract}
Let $f$ and $g$ be two regular functions on $U$ smooth affine variety. Let $\mathcal{M}$ be a regular holonomic $\mathcal{D}_{U}$-module. We are interested in the irregularity of the complex $f_{+}\left(\mathcal{M} e^{g}\right)$. More precisely, we relate the irregularity number at $c$ of the systems $\mathcal{H}^{k} f_{+}\left(\mathcal{M} e^{g}\right)$ with the characteristic cycles of the systems $\mathcal{H}^{k}(f, g)_{+}(\mathcal{M})$.
\end{abstract}

\section{$\S 1$. Introduction}

- Let $U$ be a smooth affine variety over $\mathbb{C}$ and $g: U \rightarrow \mathbb{C}$ be a regular function on $U$. We denote by $\mathcal{O}_{U}$ the sheaf of regular functions on $U$ and by $\mathcal{D}_{U}$ the sheaf of algebraic differential operators on $U$.

Let $\mathcal{M}$ be a regular holonomic $\mathcal{D}_{U}$-module. We denote by $\mathcal{M} e^{g}$ the $\mathcal{D}_{U^{-}}$ module obtained from $\mathcal{M}$ by twisting by $e^{g}$. If $\nabla$ is the connection defined by the $\mathcal{D}_{U}$-module structure of $\mathcal{M}, \nabla+d g$ is the one associated with $\mathcal{M} e^{g}$. Although $\mathcal{M}$ is regular, $\mathcal{M} e^{g}$ is not regular in general. Here, regular means that there exists a smooth compactification $X$ of $U$ and an extension of $\mathcal{M} e^{g}$ as $\mathcal{D}_{X}$-module which is regular holonomic on $X$. In [10], C. Sabbah describes a comparison theorem for these $\mathcal{D}$-modules twisted by an exponential. This theorem gives a relation between the irregularity complex of $\mathcal{M} e^{g}$ (see [6]) and some topological data given by $g$ and $\mathcal{M}$.

In this paper, we consider two regular functions $f, g: U \rightarrow \mathbb{C}$. We are interested in the irregularity of the cohomology modules of the direct image by $f$ of a $\mathcal{D}_{U}$-module, $\mathcal{M} e^{g}$, where $\mathcal{M}$ is regular and holonomic.

Communicated by M. Kashiwara. Received June 28, 2005. Revised September 27, 2005.

2000 Mathematics Subject Classification(s): 32C38, 35B40.

*Universität Mannheim, Institut für Mathematik, A5, 6, 68131 Mannheim, Germany.

e-mail: celine.roucairol@uni-mannheim.de 
- In Section 2, we recall the definitions of a $\mathcal{D}$-module twisted by an exponential of a meromorphic function. We will need the definition in the case of meromorphic function during the proof of the main theorem.

Then, we will consider the case where $\mathcal{M}$ is the sheaf of regular function $\mathcal{O}_{U}$. According to [4], the system $\mathcal{H}^{k}\left(f_{+}\left(\mathcal{O}_{U} e^{g}\right)\right)$ extends vector bundle with flat holomorphic connection such that the generic fiber of the sheaf of their horizontal sections is canonically isomorphic to the cohomology group $H_{\phi_{t}}^{k+n-1}\left(f^{-1}(t)^{a n}, \mathbb{C}\right)$, where $\phi_{t}$ is the family of closed subsets of $f^{-1}(t)$ on which $e^{-g}$ is rapidly decreasing. Using this result, we will motivate the study of the irregularity of the systems $\mathcal{H}^{k} f_{+}\left(\mathcal{O}_{U} e^{g}\right)$ by observations on some integrals.

- The main theorem of this paper gives us a formula for the irregularity number of the systems $\mathcal{H}^{k}\left(f_{+}\left(\mathcal{M} e^{g}\right)\right)$ at finite distance and at infinity.

In the case where $f$ and $g$ are two polynomials in two variables which are algebraically independents and $\mathcal{M}=\mathcal{O}_{\mathbb{C}^{2}}$, the complex $f_{+}\left(\mathcal{O}_{\mathbb{C}^{2}} e^{g}\right)$ is concentrated in degree 0 except at a finite number of points (see [9]). Then, the irregularity number at a point $c \in \mathbb{C} \cup\{\infty\}$ of the system $\mathcal{H}^{0} f_{+}\left(\mathcal{O}_{\mathbb{C}^{2}} e^{g}\right)$ can be expressed in terms of some geometric data associated with $f$ and $g$ (see [9]).

In this paper, we calculate the irregularity number at $c \in \mathbb{C} \cup\{\infty\}$ of the systems $\mathcal{H}^{k}\left(f_{+}\left(\mathcal{M} e^{g}\right)\right)$ with the help of the characteristic cycle of the systems $\mathcal{H}^{k}(f, g)_{+}(\mathcal{M})$, in the general case where $f$ and $g$ are any regular functions.

In the following, we identify $\mathbb{C} \cup\{\infty\}$ with $\mathbb{P}^{1}$. Let $i$ be the inclusion of $\mathbb{C}^{2}$ in $\mathbb{P}^{1} \times \mathbb{P}^{1}$. Let $c \in \mathbb{P}^{1}$ and $V=V_{1} \times V_{2} \subset \mathbb{P}^{1} \times \mathbb{P}^{1}$ a neighbourhood of $(c, \infty)$.

Let $C \operatorname{ch}(c, k)$ be the characteristic cycle of $\mathcal{H}^{k} i_{+}(f, g)_{+}(\mathcal{M})$ in the neighbourhood $V$ :

$C c h(c, k)=m T_{V}^{*} V+m^{\prime} T_{(c, \infty)}^{*} V+m^{\prime \prime} T_{\{c\} \times V_{2}}^{*} V+m^{\prime \prime \prime} T_{V_{1} \times\{\infty\}}^{*} V+\sum m_{l} T_{Z_{l}}^{*} V$, where $Z_{l}$ are some germs of irreducible curves in a neighbourhood of $(c, \infty)$ distinct from $V_{1} \times\{\infty\}$ and $\{c\} \times V_{2}$.

Theorem 1.1. The irregularity number of $\mathcal{H}^{k} f_{+}\left(\mathcal{M} e^{g}\right)$ at $c$ is equal to $\sum_{l} m_{l} I_{(c, \infty)}\left(Z_{l}, \mathbb{P}^{1} \times\{\infty\}\right)$, where $I_{(c, \infty)}\left(Z_{l}, \mathbb{P}^{1} \times\{\infty\}\right)$ is the intersection multiplicity of $Z_{l}$ and $\mathbb{P}^{1} \times\{\infty\}$ at $(c, \infty)$.

- The theorem of commutation between the irregularity functor and the direct image functor ([6]) allows us to rephrasing Theorem 1.1 in terms of an irregularity complex of a regular holonomic $\mathcal{D}$-module twisted by an exponential (cf. Lemma 3.2).

Then, using the comparison theorem of [10], we are led to calculate the Euler characteristic of a germ of a complex of nearby cycles. 


\section{§2. The Complex $f_{+}\left(\mathcal{M} e^{g}\right)$}

\section{$\S 2.1$. Regular holonomic $\mathcal{D}$-modules twisted by an exponential}

Let $X$ be a smooth algebraic variety over $\mathbb{C}$.

We identify $\mathbb{P}^{1}$ with $\mathbb{C} \cup\{\infty\}$. Let $h: X \rightarrow \mathbb{P}^{1}$ be a meromorphic function.

Definition 2.1. We define the $\mathcal{D}_{X^{-}}$module $\mathcal{O}_{X}\left[* h^{-1}(\infty)\right] e^{h}$ as a $\mathcal{D}_{X^{-}}$ module which is isomorphic to $\mathcal{O}_{X}\left[* h^{-1}(\infty)\right]$ as $\mathcal{O}_{X}$-module. The original connection $\nabla$ on $\mathcal{O}_{X}\left[* h^{-1}(\infty)\right]$ is replaced with the connection $\nabla+d h$ on $\mathcal{O}_{X}\left[* h^{-1}(\infty)\right] e^{h}$.

Let $\mathcal{M}$ be a holonomic $\mathcal{D}_{X}$-module.

Definition 2.2. We define the $\mathcal{D}_{X^{-}}$module $\mathcal{M}\left[* h^{-1}(\infty)\right] e^{h}$ as the $\mathcal{D}_{X^{-}}$ module $\mathcal{M} \otimes_{\mathcal{O}_{X}} \mathcal{O}_{X}\left[* h^{-1}(\infty)\right] e^{h}$.

Remark. $\quad \mathcal{O}_{X}\left[* h^{-1}(\infty)\right] e^{h}$ is the direct image by an open immersion of a vector bundle with integrable connection. Then, it is a holonomic $\mathcal{D}_{X}$-module as algebraic direct image of a holonomic $\mathcal{D}$-module.

$\mathcal{M}\left[* h^{-1}(\infty)\right] e^{h}$ is a holonomic left $\mathcal{D}_{X}$-module as tensor product of two holonomic left $\mathcal{D}_{X}$-modules (cf. Theorem 4.6 of [2]).

We have analogous definitions in the analytic case. We just have to transpose in the analytic setting.

\section{§2.2. On the solutions of the systems $\mathcal{H}^{k}\left(f_{+}\left(\mathcal{O}_{U} e^{g}\right)\right)$}

The generic fiber of the sheaf of horizonal sections of $\mathcal{H}^{k}\left(f_{+}\left(\mathcal{O}_{U} e^{g}\right)\right)$ can be describe as follows:

Theorem 2.1 ([4]). There exists a finite subset $\Sigma \subset \mathbb{C}$ such that

- $\mathcal{H}^{k}\left(f_{+}\left(\mathcal{O}_{U} e^{g}\right)\right)_{\mid \mathbb{C} \backslash \Sigma}$ is a vector bundle with flat holomorphic connection.

- For all $t \in \mathbb{C} \backslash \Sigma, i_{t}^{+} \mathcal{H}^{k-n+1}\left(f_{+}\left(\mathcal{O}_{U} e^{g}\right)\right) \simeq H_{\phi_{t}}^{k}\left(\left(f^{-1}(t)\right)^{\text {an }}, \mathbb{C}\right)$, where $i_{t}$ is the inclusion of $\{t\}$ in $\mathbb{C}$ and $\phi_{t}$ is the family of closed subsets of $f^{-1}(t)$ on which $e^{-g}$ is rapidly decreasing.

More precisely, the family $\phi_{t}$ is defined as follow. Let $\pi: \widetilde{\mathbb{P}^{1}} \rightarrow \mathbb{P}^{1}$ be the oriented real blow-up of $\mathbb{P}^{1}$ at infinity. $\widetilde{\mathbb{P}^{1}}$ is diffeomorphic to $\mathbb{C} \cup S^{1}$, where $S^{1}$ is the circle of directions at infinity. $A$ is in $\phi_{t}$ if $A$ is a closed subset of $f^{-1}(t)$ and the closure of $g(A)$ in $\mathbb{C} \cup S^{1}$ intersects $S^{1}$ in $]-\frac{\pi}{2}, \frac{\pi}{2}[$. 
Let us motivate the study of this complex by observations on some integrals. Concerning Gauss-Manin systems, we can express their solutions as period integrals of the type $\int_{\gamma(t)} w_{\mid f^{-1}(t)}$, where $\gamma(t)$ is an horizontal family of cycles in the fibres $f^{-1}(t)$ and $w$ is a relative algebraic differential form. As the Gauss-Manin connection is regular, these integrals have moderate growth in the neighbourhood of their singularities. In our case, some solutions can also be expressed as integrals.

Let $\Psi_{t}$ be the family of closed subsets $A$ of $f^{-1}(t)$ such that for all $R$ big enough, $A \backslash g^{-1}(\{t \in \mathbb{C} \mid R e(-t)>R\})$ is compact. We consider the complex of semi-algebraic chains with support in $\Psi_{t}$ (see [8]). We denote by $H_{k, \Psi_{t}}\left(f^{-1}(t)^{a n}, \mathbb{C}\right)$ the $k$-th homology group associated with this complex. We can now integrate forms in $H_{\Phi_{t}}^{k}\left(f^{-1}(t)^{a n}, \mathbb{C}\right)$ on cycles in $H_{k, \Psi_{t}}\left(f^{-1}(t)^{a n}, \mathbb{C}\right)$.

According to Theorem 1.4 of [1], since $f$ is a submersion outside $\Sigma$, we have an isomorphism

$$
\mathcal{H}^{k-n+1}\left(f_{+}\left(\mathcal{O}_{U} e^{g}\right)\right)_{\mid \mathbb{C} \backslash \Sigma} \simeq R^{k} f_{*}\left(D R_{\mathbb{C}^{n} / \mathbb{C}}\left(\mathcal{O}_{U}\right) e^{g}\right)_{\mid \mathbb{C} \backslash \Sigma}
$$

Thus, we can extend the integration defined before to a form $w e_{\mid f^{-1}(t)}^{g}$, where $w$ is a relative algebraic differential form. Indeed, by the definition of $\Psi_{t}, e^{g}$ is rapidly decreasing on the cycles and semi-algebraic chains with support in $\Psi_{t}$ behave well at infinity.

In this way, to $\gamma(t)$, horizontal family of cycles in $H_{k, \Psi_{t}}\left(f^{-1}(t)^{a n}, \mathbb{C}\right)$, we can associate a solution of the $\mathcal{D}_{\mathbb{C} \backslash \Sigma}$-module $\mathcal{H}^{k-n+1}\left(f_{+}\left(\mathcal{O}_{U} e^{g}\right)\right)_{\mid \mathbb{C} \backslash \Sigma}$. It is a morphism $\alpha$ of $\mathcal{D}_{\mathbb{C} \backslash \Sigma^{-m}}$ modules defined by $\alpha\left(\left[w e^{g}\right]\right)=\int_{\gamma(t)} w e_{\mid f^{-1}(t)}^{g}$.

The study of the irregularity of the systems $\mathcal{H}^{k-n+1}\left(f_{+}\left(\mathcal{O}_{U} e^{g}\right)\right)$ gives us informations about the growth of these integrals in the neighbourhood of their singularities.

\section{$\S 3 . \quad$ On the Irregularity of the Complex $f_{+}\left(\mathcal{M} e^{g}\right)$}

In the following, we will identify $\mathbb{C} \cup\{\infty\}$ with $\mathbb{P}^{1}$ and we consider the canonical immersion $j: \mathbb{C} \rightarrow \mathbb{P}^{1}$. Let us fix $k \in \mathbb{Z}$ and $c \in \mathbb{P}^{1}$.

We are interested in the number $I R_{c, k}$, it being the irregularity number at $c \in \mathbb{P}^{1}$ of the system $\mathcal{H}^{k} j_{+} f_{+}\left(\mathcal{M} e^{g}\right)$.

The first step of the proof of Theorem 1.1 consists in rephrasing it using

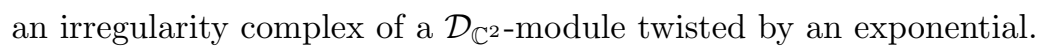

For the definition of irregularity complex along an hypersurface, we refer the reader to [6] and [7]. We adopt the following notations. If $\mathfrak{M}$ is a complex of $\mathcal{D}_{X}$-modules and $Z$ is an hypersurface of $X$, we denote by $I R_{Z}(\mathfrak{M})$ the irregu- 
larity complex of $\mathfrak{M}$ along $Z$. For simplicity of notations, we write $I R_{Z}^{k}(\mathfrak{M})$ instead of $\mathcal{H}^{k}\left(I R_{Z}(\mathfrak{M})\right)$.

According to [5], the irregularity number $I R_{c, k}$ is equal to the dimension of the $\mathbb{C}$-vector space $I R^{0}\left(\mathcal{H}^{k} j_{+} f_{+}\left(\mathcal{M} e^{g}\right)\right)_{c}$.


of rephrasing Theorem 1.1, we need the following lemma:

Lemma 3.1. Let $\pi_{2}: \mathbb{P}^{1} \times \mathbb{P}^{1} \rightarrow \mathbb{P}^{1}$ be the second projection and $D=$ $\left(\mathbb{P}^{1} \times \mathbb{P}^{1}\right) \backslash i\left(\mathbb{C}^{2}\right)$.

$$
I R_{c}\left(\mathcal{H}^{k} j_{+} f_{+}\left(\mathcal{M} e^{g}\right)\right)_{c}=I R_{\{c\} \times \mathbb{P}^{1}}\left(\mathcal{H}^{k}\left(\mathcal{N}^{\bullet}\right)[* D] e^{\pi_{2}}\right)_{(c, \infty)}[+1] .
$$

Proof. - Reduction to the case of the two projections.

Let $p_{1}: \mathbb{C}^{2} \rightarrow \mathbb{C}$ and $p_{2}: \mathbb{C}^{2} \rightarrow \mathbb{C}$ be the two canonical projections.

As $f=p_{1} \circ(f, g)$, we have $f_{+}\left(\mathcal{M} e^{g}\right)=p_{1+}(f, g)_{+}\left(\mathcal{M} e^{g}\right)$. Moreover, $(f, g)_{+}\left(\mathcal{M} e^{g}\right)=(f, g)_{+}(\mathcal{M}) e^{p_{2}}$.

Finally, we obtain that $f_{+}\left(\mathcal{M} e^{g}\right) \simeq p_{1+}\left((f, g)_{+}(\mathcal{M}) e^{p_{2}}\right)$.

- In this paragraph, we denote by ${ }^{p} \mathcal{H}$ the perverse cohomology. According to Corollary 2-1-8 of [6], we have:

$$
I R_{c}\left(\mathcal{H}^{k} j_{+} f_{+}\left(\mathcal{M} e^{g}\right)\right)={ }^{p} \mathcal{H}^{k} I R_{c}\left(j_{+} f_{+}\left(\mathcal{M} e^{g}\right)\right) .
$$

Consider the following diagrams:
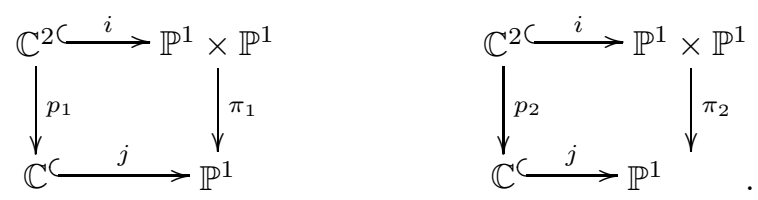

Then,

$$
\begin{aligned}
I R_{c}\left(j_{+} f_{+}\left(\mathcal{M} e^{g}\right)\right) & =I R_{c}\left(j_{+} p_{1+}\left((f, g)_{+}(\mathcal{M}) e^{p_{2}}\right)\right) \\
& =I R_{c}\left(\pi_{1+} i_{+}\left((f, g)_{+}(\mathcal{M}) e^{p_{2}}\right)\right) \\
& =I R_{c}\left(\pi_{1+} i_{+}\left(\mathcal{N}^{\bullet}[* D] e^{\pi_{2}}\right)\right) .
\end{aligned}
$$

Then, $I R_{c}\left(\mathcal{H}^{k} j_{+} f_{+}\left(\mathcal{M} e^{g}\right)\right)={ }^{p} \mathcal{H}^{k} I R_{c}\left(\pi_{1+} i_{+}\left(\mathcal{N}^{\bullet}[* D] e^{\pi_{2}}\right)\right)$.

- According to Proposition 3-6-4 of [7], the irregularity functor commutes with the direct image functor. Thus:

$$
\begin{aligned}
I R_{c}\left(\pi_{1+} i_{+}\left(\mathcal{N}^{\bullet}[* D] e^{\pi_{2}}\right)\right)_{c} & =\mathbb{R} \pi_{1 *} I R_{\{c\} \times \mathbb{P}^{1}}\left(\mathcal{N}^{\bullet}[* D] e^{\pi_{2}}\right)_{c}[+1] \\
& =\mathbb{R} \Gamma\left(\{c\} \times \mathbb{P}^{1}, I R_{\{c\} \times \mathbb{P}^{1}}\left(\mathcal{N}^{\bullet}[* D] e^{\pi_{2}}\right)\right)[+1]
\end{aligned}
$$


- Then, we remark that $\pi_{2}$ is holomorphic out of $(c, \infty)$ and $\mathcal{N}^{\bullet}$ is regular holonomic (direct image complex of an algebraic regular holonomic $\mathcal{D}$-module). Then, $I R_{\{c\} \times \mathbb{P}^{1}}\left(\mathcal{N}^{\bullet}[* D] e^{\pi_{2}}\right)$ has its support in $(c, \infty)$ and we have an isomorphism of complexes of vector spaces

$$
I R_{c}\left(\pi_{1+} i_{+}\left(\mathcal{N}^{\bullet}[* D] e^{\pi_{2}}\right)\right)_{c}=I R_{\{c\} \times \mathbb{P}^{1}}\left(\mathcal{N}^{\bullet}[* D] e^{\pi_{2}}\right)_{(c, \infty)}[+1] .
$$

- We conclude that

$$
\begin{aligned}
I R_{c}\left(\mathcal{H}^{k} j_{+} f_{+}\left(\mathcal{M} e^{g}\right)\right)_{c} & ={ }^{p} \mathcal{H}^{k} I R_{c}\left(\pi_{1+} i_{+}\left(\mathcal{N}^{\bullet}[* D] e^{\pi_{2}}\right)\right)_{c} \\
& ={ }^{p} \mathcal{H}^{k} I R_{\{c\} \times \mathbb{P}^{1}}\left(\mathcal{N}^{\bullet}[* D] e^{\pi_{2}}\right)_{(c, \infty)}[+1] \\
& =I R_{\{c\} \times \mathbb{P}^{1}}\left(\mathcal{H}^{k}\left(\mathcal{N}^{\bullet}[* D] e^{\pi_{2}}\right)\right)_{(c, \infty)}[+1] \\
& =I R_{\{c\} \times \mathbb{P}^{1}}\left(\mathcal{H}^{k}\left(\mathcal{N}^{\bullet}\right)[* D] e^{\pi_{2}}\right)_{(c, \infty)}[+1]
\end{aligned}
$$

Now, we can rephrase Theorem 1.1.

Let us choose some local coordinates $(x, z)$ of $\mathbb{P}^{1} \times \mathbb{P}^{1}$ in a neighbourhood of $(c, \infty)$ such that:

- $(c, \infty)$ has for coordinates $(0,0)$,

- $\{c\} \times \mathbb{P}^{1}$ has equation $x=0$ in a neighbourhood of $(c, \infty)$,

- $\mathbb{P}^{1} \times\{\infty\}$ has equation $z=0$ in a neighbourhood of $(c, \infty)$.

In these coordinates, $\pi_{2}$ is equal to $\frac{1}{z}$ in a neighbourhood of $(c, \infty)$. Then, according to Lemma 3.1, we are led to prove the following lemma:

Lemma 3.2. Let $\mathfrak{M}$ be a holonomic regular $\mathcal{D}_{\mathbb{C}^{2} \text {-module. }}$ We denote the characteristic cycle of $\mathfrak{M}$ in a neighbourhood of $(0,0)$ by:

$$
C \operatorname{ch}(\mathfrak{M})=m T_{\mathbb{C}^{2}}^{*} \mathbb{C}^{2}+m^{\prime} T_{(c, \infty)}^{*} \mathbb{C}^{2}+m^{\prime \prime} T_{x=0}^{*} \mathbb{C}^{2}+m^{\prime \prime \prime} T_{z=0}^{*} \mathbb{C}^{2}+\sum m_{l} T_{Z_{l}}^{*} \mathbb{C}^{2},
$$

where $Z_{l}$ are some germs of irreducible curves in a neighbourhood of $(0,0)$ distinct from $x=0$ and $z=0$.

Then,

$$
\chi\left(I R_{x=0}\left(\mathfrak{M}\left[\frac{1}{z}\right] e^{\frac{1}{z}}\right)_{(0,0)}=-\sum_{l} m_{l} I_{(c, \infty)}\left(Z_{l},\{z=0\}\right) .\right.
$$




\section{§4. Proof of Lemma 3.2}

We break up the proof of Lemma 3.2 in three steps:

Lemma 4.1. $\quad \chi\left(I_{x=0}\left(\mathfrak{M}\left[\frac{1}{z}\right] e^{\frac{1}{z}}\right)_{(0,0)}\right)=\chi\left(I R_{z=0}\left(\mathfrak{M}\left[\frac{1}{x z}\right] e^{\frac{1}{z}}\right)_{(0,0)}\right)$.

We denote by $\Psi_{z}\left(\mathfrak{M}\left[\frac{1}{x}\right]\right)$ the complex of nearby cycles of $\mathfrak{M}\left[\frac{1}{x}\right]$ relative to $z$. It is a complex of constructible sheaves on $\mathbb{C} \times\{0\}$ defined as follows.

Let $\eta$ small enough. We denote by $\widetilde{D^{*}(0, \eta)}$ the universal covering of $D^{*}(0, \eta)$. Let $(E, \pi, \tilde{z})$ be the fiber product over $D^{*}(0, \eta)$ of $\mathbb{C} \times D^{*}(0, \eta)$ and $\widetilde{D^{*}(0, \eta)}$. Then, we have the following diagram:

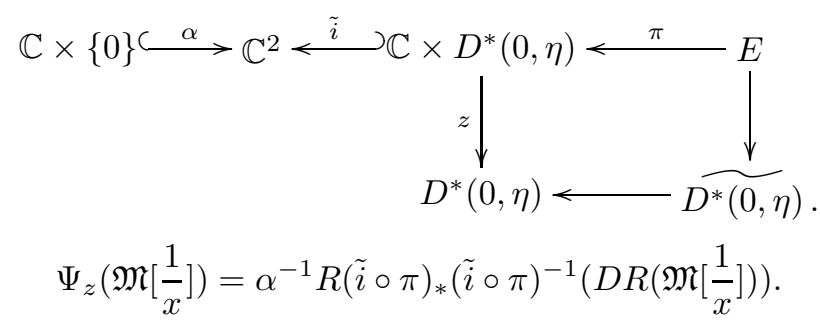

Lemma 4.2. $\quad \chi\left(I R_{z=0}\left(\mathfrak{M}\left[\frac{1}{x z}\right] e^{\frac{1}{z}}\right)_{(0,0)}\right)=\chi\left(\Psi_{z}\left(\mathfrak{M}\left[\frac{1}{x}\right]\right)_{(0,0)}\right)$.

Lemma 4.3. $\quad \chi\left(\Psi_{z}\left(\mathfrak{M}\left[\frac{1}{x}\right]\right)_{(0,0)}\right)=-\sum_{l} m_{l} I_{(c, \infty)}\left(Z_{l}, \mathbb{P}^{1} \times\{\infty\}\right)$.

Proof of Lemma 4.1. Let us first show that

$$
I R_{x=0}\left(\mathfrak{M}\left[\frac{1}{z}\right] e^{\frac{1}{z}}\right)=R \Gamma_{x=0}\left(I R_{z=0}\left(\mathfrak{M}\left[\frac{1}{x z}\right] e^{\frac{1}{z}}\right)\right) .
$$

Let $\eta$ be the inclusion of $\mathbb{C} \times \mathbb{C}^{*}$ in $\mathbb{C}^{2}$. By definition,

$$
\begin{aligned}
I_{z=0}\left(\mathfrak{M}\left[\frac{1}{x z}\right] e^{\frac{1}{z}}\right) & =\operatorname{cone}\left(\operatorname{DR}\left(\mathfrak{M}\left[\frac{1}{x z}\right] e^{\frac{1}{z}}\right) \rightarrow R \eta_{*} \eta^{-1}\left(D R\left(\mathfrak{M}\left[\frac{1}{x z}\right] e^{\frac{1}{z}}\right)\right)\right) \\
& =\operatorname{cone}\left(\operatorname{DR}\left(\mathfrak{M}\left[\frac{1}{x z}\right] e^{\frac{1}{z}}\right) \rightarrow R \eta_{*}\left(D R\left(\mathfrak{M}\left[\frac{1}{x}\right]\right)_{\mid \mathbb{C} \times \mathbb{C}^{*}}\right)\right) .
\end{aligned}
$$

Now, consider the following diagram:

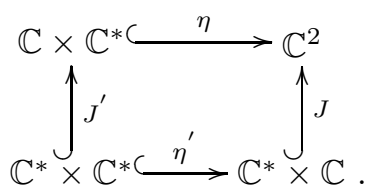


As $\mathfrak{M}$ is regular, we have:

$$
\begin{aligned}
R \eta_{*}\left(D R\left(\mathfrak{M}\left[\frac{1}{x}\right]\right)_{\mid \mathbb{C} \times \mathbb{C}^{*}}\right) & =R \eta_{*} R J_{*}^{\prime}\left(D R(\mathfrak{M})_{\mid \mathbb{C}^{*} \times \mathbb{C}^{*}}\right) \\
& =R J_{*} R \eta_{*}^{\prime}\left(D R(\mathfrak{M})_{\mid \mathbb{C}^{*} \times \mathbb{C}^{*}}\right)
\end{aligned}
$$

But $R \Gamma_{x=0} R J_{*}=0$. Then:

$$
\begin{aligned}
R \Gamma_{x=0}\left(I R_{z=0}\left(\mathfrak{M}\left[\frac{1}{x z}\right] e^{\frac{1}{z}}\right)\right) & =R \Gamma_{x=0}\left(D R\left(\mathfrak{M}\left[\frac{1}{x z}\right] e^{\frac{1}{z}}\right)\right) \\
& =I R_{x=0}\left(\mathfrak{M}\left(\frac{1}{z}\right] e^{\frac{1}{z}}\right)
\end{aligned}
$$

by definition of irregularity complex.

Then, we are led to prove that the complexes $R \Gamma_{x=0}\left(I R_{z=0}\left(\mathfrak{M}\left[\frac{1}{x z}\right] e^{\frac{1}{z}}\right)\right)$ and $I R_{z=0}\left(\mathfrak{M}\left[\frac{1}{x z}\right] e^{\frac{1}{z}}\right)$ have the same characteristic function at $(0,0)$.

Using the following distinguished triangle,

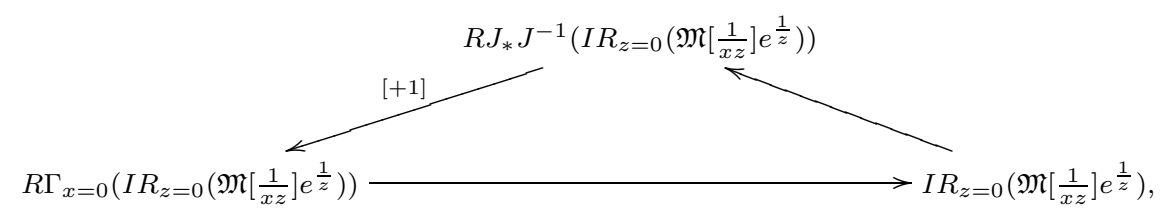

it is sufficient to show that the characteristic function on $\{x=0\}$ of the complex $R J_{*} J^{-1}\left(I R_{z=0}\left(\mathfrak{M}\left[\frac{1}{x z}\right] e^{\frac{1}{z}}\right)\right)$ is zero.

Now, if $\mathcal{F}$ is a constructible sheaf on $X$ and $P \in\{x=0\}$,

$$
\chi\left(\left(R J_{*} J^{-1} \mathcal{F}\right)_{P}\right)=\chi\left(\left(\mathbb{D}\left(J_{!} J^{-1} \mathbb{D} \mathcal{F}\right)\right)_{P}\right)=\chi\left(\left(J_{!} J^{-1} \mathbb{D} \mathcal{F}\right)_{P}\right)=0,
$$

where $\mathbb{D}$ is the Verdier duality (see [11]).

Proof of Lemma 4.2. This is a particular case of a result of C. Sabbah (cf. Corollary 5-2 of [10]).

Proof of Lemma 4.3. Denote by $\mathcal{C} \bullet$ the complex $\Psi_{z}\left(\mathfrak{M}\left[\frac{1}{x}\right]\right)$. By definition, $\mathcal{C}_{(0,0)}^{\bullet}=R(\tilde{i} \circ \pi)_{*}(\tilde{i} \circ \pi)^{-1}\left(D R\left(\mathfrak{M}\left[\frac{1}{x}\right]\right)\right)_{(0,0)}$.

- Then, for all $k \in \mathbb{Z}$,

$$
\mathcal{H}^{k} \mathcal{C}_{(0,0)}^{\bullet}=\underset{(0,0) \in U \text { open }}{\text { indlim }} \mathbb{R}^{k} \Gamma\left(U, R(\tilde{i} \circ \pi)_{*}(\tilde{i} \circ \pi)^{-1}\left(D R\left(\mathfrak{M}\left[\frac{1}{x}\right]\right)\right)\right) .
$$

As $\left\{D\left(0, \eta_{1}\right) \times D\left(0, \eta_{2}\right)\right\}_{\eta_{1}, \eta_{2}}$ is a fundamental system of neighbourhoods of $(0,0)$, we have

$$
\mathcal{H}^{k} \mathcal{C}_{(0,0)}^{\bullet} \underset{\eta_{1}, \eta_{2}}{\operatorname{indlim}} \mathbb{R}^{k} \Gamma\left(D\left(0, \eta_{1}\right) \times D\left(0, \eta_{2}\right), R(\tilde{i} \circ \pi)_{*}(\tilde{i} \circ \pi)^{-1}\left(D R\left(\mathfrak{M}\left[\frac{1}{x}\right]\right)\right)\right)
$$


- Let $\Sigma$ be a Whitney stratification associated with the constructible sheaf $D R\left(\mathfrak{M}\left[\frac{1}{x}\right]\right)$. Then, for $\eta_{1}$ and $\eta_{2}$ small enough, there exists a homotopy equivalence $p:(\tilde{i} \circ \pi)^{-1}\left(D\left(0, \eta_{1}\right) \times D\left(0, \eta_{2}\right)\right) \rightarrow D\left(0, \eta_{1}\right) \times\{\tilde{\eta}\}$ compatible with $\Sigma$. Thus, $\mathcal{H}^{k} \mathcal{C}_{(0,0)}^{\bullet}=\underset{\eta_{1}, \eta_{2}}{\operatorname{indlim}} \mathbb{R} \Gamma\left(D\left(0, \eta_{1}\right) \times\{\tilde{\eta}\}, D R\left(\mathfrak{M}\left[\frac{1}{x}\right]\right)\right)$.

- Now, as $\mathfrak{M}$ is regular, $D R\left(\mathfrak{M}\left[\frac{1}{x}\right]\right)=R J_{*} J^{-1}(D R(\mathfrak{M}))$. Then,

$$
\begin{aligned}
\mathcal{H}^{k} \mathcal{C}_{(0,0)}^{\bullet} & =\underset{\substack{\eta_{1}, \eta_{2} \\
\operatorname{indim}}}{\operatorname{Ro}} \Gamma\left(D\left(0, \eta_{1}\right) \times\{\tilde{\eta}\}, R J_{*} J^{-1}(D R(\mathfrak{M}))\right) \\
& =\underset{\eta_{1}, \eta_{2}}{\operatorname{indlim}} \mathbb{R} \Gamma\left(D^{*}\left(0, \eta_{1}\right) \times\{\tilde{\eta}\}, J^{-1}(D R(\mathfrak{M}))\right) .
\end{aligned}
$$

- Let fix $\eta_{1}$ and $\tilde{\eta}$ small enough such that the singular support of $\mathfrak{M}$ in $D^{*}\left(0, \eta_{1}\right) \times\{\tilde{\eta}\}$ is a finite number of points. Denote by $P_{1}, \ldots, P_{s}$ these points. They are the intersection points of $D^{*}\left(0, \eta_{1}\right) \times\{\tilde{\eta}\}$ and $\cup Z_{l}$. As $D R(\mathfrak{M})_{\mid D^{*}\left(0, \eta_{1}\right) \times\{\tilde{\eta}\}}$ is a complex of constructible sheaves with respect to the stratification $\left\{D^{*}\left(0, \eta_{1}\right) \times\{\tilde{\eta}\} \backslash\left\{P_{1}, \ldots, P_{l}\right\}, P_{1}, \ldots, P_{l}\right\}$, the Euler characteristic of $\mathbb{R} \Gamma\left(D^{*}\left(0, \eta_{1}\right) \times\{\tilde{\eta}\}, J^{\prime-1}(D R(\mathfrak{M}))\right.$ is equal to:

$$
\chi\left(\mathbb{R} \Gamma\left(D^{*}\left(0, \eta_{1}\right) \times\{\tilde{\eta}\} \backslash\left\{P_{1}, \ldots, P_{l}\right\}, D R(\mathfrak{M})\right)\right)+\sum_{i=1}^{l} \chi\left(D R(\mathfrak{M})_{P_{i}}\right) .
$$

Then, according to the index theorem of Kashiwara (cf. [3]),

$$
\begin{aligned}
\chi & \mathbb{R} \Gamma\left(D^{*}\left(0, \eta_{1}\right) \times\{\tilde{\eta}\}, J^{\prime}-1(D R(\mathfrak{M}))\right) \\
& =r k(\mathfrak{M}) \sum_{l} I_{(0,0)}\left(Z_{l},\{z=0\}\right)+\sum_{l}\left(r k(\mathfrak{M})-m_{l}\right) I_{(0,0)}\left(Z_{l},\{z=0\}\right) \\
& =-\sum_{l} m_{l} I_{(0,0)}\left(Z_{l},\{z=0\}\right) .
\end{aligned}
$$

Remark. If $f$ and $g$ are two polynomials in two variables, we can compare Theorem 1.1 and Theorem 1 of [9]. Let us recall this theorem:

Let $\mathbb{X}$ be a smooth projective compactification of $\mathbb{C}^{2}$ such that there exists $F, G: \mathbb{X} \rightarrow \mathbb{P}^{1}$, two meromorphic maps, which extend $f$ and $g$. Let us denotes by $D$ the divisor $\mathbb{X} \backslash \mathbb{C}^{2}$. Let $\Gamma$ be the critical locus of $(F, G)$.

Let $c \in \mathbb{P}^{1}$. We denote by $\Delta_{1}$ the cycle in $\mathbb{P}^{1} \times \mathbb{P}^{1}$ which is the closure in $\mathbb{P}^{1} \times \mathbb{P}^{1}$ of $(F, G)(\Gamma) \cap\left(\mathbb{C}^{2} \backslash\{c\} \times \mathbb{C}\right)$, where the image is counted with multiplicity and by $\Delta_{2}$ the cycle in $\mathbb{P}^{1} \times \mathbb{P}^{1}$ which is the closure in $\mathbb{P}^{1} \times \mathbb{P}^{1}$ of $(F, G)(D) \cap\left(\mathbb{C}^{2} \backslash\{c\} \times \mathbb{C}\right)$, where the image is counted with multiplicity.

Theorem 4.1. If $f$ and $g$ are algebraically independent, the irregularity number of $\mathcal{H}^{0}\left(f_{+}\left(\mathcal{O}_{\mathbb{C}^{2}} e^{g}\right)\right)$ is equal to

$$
I_{(c, \infty)}\left(\Delta_{1}, \mathbb{P}^{1} \times\{\infty\}\right)+I_{(c, \infty)}\left(\Delta_{2}, \mathbb{P}^{1} \times\{\infty\}\right) .
$$


Then, we can prove that the germs $Z_{l}$ of irreducible curves in Theorem 1.1 are the germs at $(c, \infty)$ of the irreducible branches of $\Delta_{1} \cup \Delta_{2}$. The multiplicity $m_{l}$ of $i_{+}(f, g)_{+}\left(\mathcal{O}_{\mathbb{C}^{2}}\right)$ on $T_{Z_{l}}^{*} V$ are the multiplicity of $Z_{k}$ in $\Delta_{1} \cup \Delta_{2}$.

\section{References}

[1] Dimca, A., Maaref, F., Sabbah, C. and Saito, M., Dwork cohomology and algebraic $\mathcal{D}$-modules, Math. Ann., 318 (2000), 107-125.

[2] Kashiwara, M., Systems of microdifferential equation, Progress in Math., 34, Birkhäuser, Boston, 1983.

[3] Kashiwara, M., Index theorem for constructible sheaves, in: systèmes différentiels et singularités, Astérisque, 130 (1985), 193-209.

[4] Maaref, F., Sur un analogue irrégulier de la connexion de Gauss-Manin, Ann. Fac. Sci. Toulouse Math., 8 (1999), 117-124.

[5] Malgrange, B., Sur les points singuliers des équations différentielles, Enseignement Math., 20 (1974), 147-176.

[6] Mebkhout, Z., Le théorème de positivité de l'irrégularité pour les $\mathcal{D}_{X}$-modules, Grothendieck festschrift, III, Progress in Math., 88 (1990), 84-131.

[7] Mebkhout, Z., Le théorème de positivité, le théorème de comparaison, le théorème d'existence de Riemann, séminaire et congrès, 8 (2004), 165-307.

[8] Pham, F., La descente des cols par les onglets de Lefschetz avec vue sur Gauss-Manin, in: systèmes différentiels et singularités, Astérisque, 130 (1985), 11-47.

[9] Roucairol, C., Irregularity of an analogue of the Gauss-Manin systems, ArXiv:AG/0505075, 2005.

[10] Sabbah, C., On the comparaison theorem for elementary irregular $\mathcal{D}$-modules, Nagoya Math. J., 141 (1996), 107-124.

[11] Verdier, J. L., Dualité de Poincaré, Séminaire Heidelberg-Strasbourg 1966-67, Publ. I.R.M.A. 3, Strasbourg, 1969. 